\title{
Comparison of the Effectiveness and Clinical Outcome of Everolimus Followed by CDK4/6 Inhibitors with the Opposite Treatment Sequence in Hormone Receptor-Positive, HER2-Negative Metastatic Breast Cancer
}

\author{
Hyehyun Jeong, Jae Ho Jeong, Jeong Eun Kim, Jin-Hee Ahn, Kyung Hae Jung, Sung-Bae Kim \\ Department of Oncology, Asan Medical Center, University of Ulsan College of Medicine, Seoul, Korea
}

Purpose In hormone receptor-positive, human epidermal growth factor receptor 2-negative metastatic breast cancer (HR+ HER2$\mathrm{MBC}$ ), the mainstay treatment options include cyclin-dependent kinase 4/6 inhibitors (CDK4/6i) and everolimus (EVE) in combination with endocrine treatment. This study aims to compare the outcomes of the following treatment sequences: CDK4/6i followed by EVE and EVE followed by CDK4/6i.

Materials and Methods Data from HR+ HER2- MBC patients treated between January 2014 and November 2020 with both CDK4/6i and EVE were retrospectively analyzed.

Results Among the 88 patients included in the study, 51 received CDK4/6i before EVE (C $\rightarrow$ E group), and 37 received EVE before $\mathrm{CDK} 4 / 6 \mathrm{i}(\mathrm{E} \rightarrow \mathrm{C}$ group) with endocrine treatment. More patients in the $\mathrm{E} \rightarrow \mathrm{C}$ group had endocrine resistance (13.7\% vs. 40.5\%), experienced palliative chemotherapy (7.8\% vs. $40.5 \%$ ), and were heavily treated (treated as $\geq 3$ rd line, $5.9 \%$ vs. $40.5 \%$ ). Median overall survival was 46.8 months in the $\mathrm{C} \rightarrow \mathrm{E}$ group and 38.9 months in the $\mathrm{E} \rightarrow \mathrm{C}$ group $(\mathrm{p}=0.151)$. Median composite progression-free survival (PFS), defined as the time from the start of the preceding regimen to disease progression on the following regimen or death, was 24.8 months in the $\mathrm{C} \rightarrow \mathrm{E}$ group vs. 21.8 months in the $\mathrm{E} \rightarrow \mathrm{C}$ group $(\mathrm{p}=0.681)$. Median PFS2/PFS1 ratio did not differ significantly between groups (0.5 in the $\mathrm{C} \rightarrow \mathrm{E}$ group, 0.6 in the $\mathrm{E} \rightarrow \mathrm{C}$ group; $\mathrm{p}=0.775)$. Ten patients (11.4\%) discontinued $\mathrm{EVE}$, and two patients (2.3\%) discontinued CDK4/6i during treatment.

Conclusion Although the CDK4/6i-based regimen should be considered as an earlier line of treatment, CDK4/6i- and EVE-based treatments can be valid options in circumstances where the other treatment had been already given.

Key words Cyclin-dependent kinase 4, Cyclin-dependent kinase 6, Protein kinase inhibitors, Everolimus, Breast neoplasms

\section{Introduction}

The combination of cyclin-dependent kinase $4 / 6$ inhibitors $(\mathrm{CDK} 4 / 6 \mathrm{i})$ with endocrine treatment has become a standard treatment in hormone receptor-positive (HR+), human epidermal growth factor receptor 2-negative (HER2) metastatic breast cancer (MBC). Three major CDK4/6i (palbociclib, ribociclib, and abemaciclib) consistently showed significant progression-free survival (PFS) benefits with favorable adverse event profiles [1-7]. Moreover, recent longterm follow-up results of pivotal phase 3 trials for CDK4 / 6ibased treatment showed an overall survival (OS) benefit [8-10], which further supports the use of the combination as a frontline choice in patients with HR+ MBC. On the other hand, everolimus (EVE), a mammalian target of rapamycin (mTOR) inhibitor, has previously shown a remarkable PFS benefit in HR+ MBC patients [11], but it did not confer a statistically significant OS benefit [12]. Based on these results,
EVE-based regimens comprise one of the major therapeutic choices for $\mathrm{HR}+\mathrm{MBC}$, and CDK4 / 6i-based regimens are preferred frontline treatments.

While these two agents comprise important parts of the management of HR+ HER2- MBC, whether their efficacy could be affected by treatment sequence or prior regimens is currently not well studied. Recent preclinical studies have demonstrated the interaction between CDK4/ 6 inhibition and activation of the phosphoinositide 3-kinase/AKT/ mTOR pathway $[13,14]$, along with the possible synergistic anti-tumor effect of CDK4/6i in combination with mTOR inhibitors in vitro $[15,16]$. However, previous pivotal studies, including PALOMA-3 [4] and MONARCH-2 [5], excluded patients treated with EVE, and few clinical studies have addressed the efficacy of EVE following CDK4/6i-based regimens or vice versa.

Therefore, this retrospective study aims to evaluate the clinical outcomes by treatment sequence of CDK4/6i- and 
EVE-based regimens, both of which are major therapeutic choices for $\mathrm{HR}+\mathrm{MBC}$ patients.

\section{Materials and Methods}

\section{Patients}

HR+ HER2- MBC patients treated with both CDK4/6iand EVE-based regimens between January 2014 and November 2020 were included in this study, irrespective of other treatments given between the two regimens.

\section{Treatment and assessment}

Patients were treated with both CDK4/6i-based (combination of CDK4/6 inhibitors and endocrine treatment) and EVE-based (combination of EVE and endocrine treatment) regimens. Tumor response was assessed by the Response Evaluation Criteria in Solid Tumors (RECIST) ver. 1.1. Endocrine sensitivity was defined as at least 24 months of adjuvant endocrine therapy before recurrence or a disease response or stabilization for at least 24 weeks of endocrine therapy in the case of advanced disease. PFS $\mathrm{C}+\mathrm{E}$ was defined as the composite PFS from the start of either CDK4/6i or EVE treatment to the date of disease progression to the following regimen or the date of death, whichever occurred first. If treatment other than CDK4/6i or EVE-based regimens was given in between, the duration of such treatment was included in the $\mathrm{PFS}_{\mathrm{C}+\mathrm{E} .}$ PFS $_{\mathrm{C}}$ indicated PFS for the CDK4/6i-based regimen, and $\mathrm{PFS}_{\mathrm{E}}$ indicated PFS for the EVE-based regimen. $\mathrm{PFS}_{1}$ and $\mathrm{PFS}_{2}$ indicated PFS to the preceding regimen (e.g. in the $\mathrm{C} \rightarrow \mathrm{E}$ group, $\mathrm{CDK} 4 / 6 \mathrm{i}$, in the $\mathrm{E} \rightarrow \mathrm{C}$ group, EVE) and to the following regimen, respectively, for CDK4/6i and EVE. OS was defined as the time from the start of either regimen to death by any cause.

\section{Statistical analysis}

Baseline characteristics were analyzed and compared by descriptive methods. Survival outcomes were estimated by the Kaplan-Meier method and compared by a log-rank test. All tests were two-sided, and a p-value less than 0.05 was considered statistically significant. Statistical analyses were performed using $\mathrm{R}$ ver. 4.0.1 ( $\mathrm{R}$ Foundation for Statistical Computing, Vienna, Austria).

\section{Results}

\section{Patients}

A total of 88 patients were included in the study. Among these, 51 patients received the $\mathrm{CDK} 4 / 6 \mathrm{i}$-based regimen before the EVE-based regimen $(\mathrm{C} \rightarrow \mathrm{E}$ group), and 37 patients received the EVE-based regimen before the CDK4/6i-based regimen ( $\mathrm{E} \rightarrow \mathrm{C}$ group) (Fig. 1). The baseline characteristics of the patients are shown in Table 1 . The proportion of patients who received either the CDK4/6i- or EVE-based regimen as a first-line treatment was higher in the $\mathrm{C} \rightarrow \mathrm{E}$ group $(64.7 \%$ vs. $10.8 \%$ ), whereas the proportion of patients who received either regimen for $\geq$ third-line treatment was higher in the $\mathrm{E} \rightarrow \mathrm{C}$ group (5.9\% vs. 40.5\%). More patients in the $\mathrm{E} \rightarrow \mathrm{C}$ group had an endocrine-resistant disease (13.7\% vs. $40.5 \%)$ and had experienced chemotherapy before either regimen was given (7.8\% vs. $40.5 \%$ ). Seventy-five percent of patients in the overall study population had visceral metastases at the start of either regimen, without significant differences between groups.

CDK4/6i-based regimens comprised palbociclib plus letrozole, palbociclib plus fulvestrant, and abemaciclib plus fulvestrant. All patients treated in combination with letrozole were included in the $\mathrm{C} \rightarrow \mathrm{E}$ group. All patients received EVE+exemestane as the EVE-based regimen. Among the

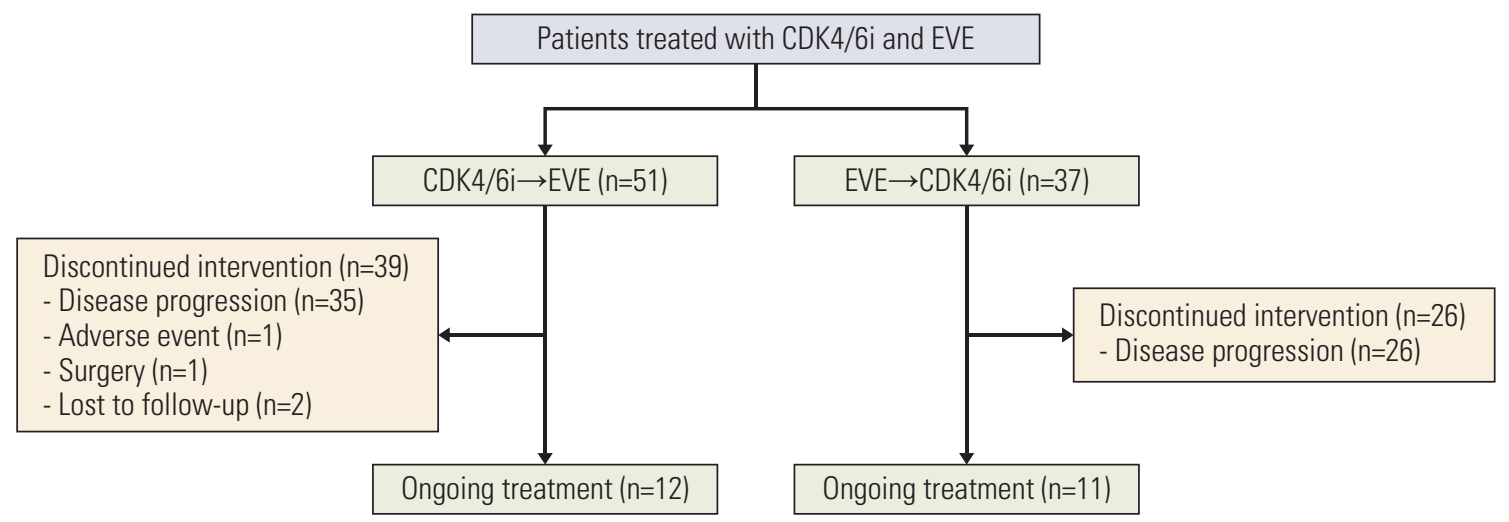

Fig. 1. CONSORT diagram. CDK4 / 6i, cyclin-dependent kinase 4/ 6 inhibitor; EVE, everolimus. 
Table 1. Baseline characteristics

\begin{tabular}{|c|c|c|c|c|}
\hline & Overall $(n=88)$ & $C \rightarrow E(n=51)$ & $E \rightarrow C(n=37)$ & p-value \\
\hline Age (yr) & $53(35-76)$ & $52(36-76)$ & $54(35-70)$ & 0.568 \\
\hline \multicolumn{5}{|l|}{ ECOG PS } \\
\hline 0 & $19(21.6)$ & $16(31.4)$ & $3(8.1)$ & 0.032 \\
\hline 1 & $46(52.3)$ & $23(45.1)$ & $23(62.2)$ & \\
\hline Unknown & $23(26.1)$ & $12(23.5)$ & $11(29.7)$ & \\
\hline \multicolumn{5}{|c|}{ Menopausal status at diagnosis of advanced disease } \\
\hline Pre/perimenopausal & $37(42.0)$ & $21(41.2)$ & $16(43.2)$ & 0.916 \\
\hline Postmenopausal & $49(55.7)$ & $29(56.9)$ & $20(54.1)$ & \\
\hline Unknown & $2(2.3)$ & $1(2.0)$ & $1(2.7)$ & \\
\hline \multicolumn{5}{|l|}{ Line of treatment } \\
\hline 1 & $37(42.0)$ & $33(64.7)$ & $4(10.8)$ & $<0.001$ \\
\hline 2 & $33(37.5)$ & $15(29.4)$ & $18(48.6)$ & \\
\hline$\geq 3$ & $18(20.5)$ & $3(5.9)$ & $15(40.5)$ & \\
\hline \multicolumn{5}{|l|}{ Purpose of most recent endocrine treatment } \\
\hline Adjuvant & $34(38.6)$ & $30(58.8)$ & $4(10.8)$ & $<0.001$ \\
\hline Palliative & $46(52.3)$ & $13(25.5)$ & $33(89.2)$ & \\
\hline No prior endocrine treatment & $8(9.1)$ & $8(15.7)$ & 0 & \\
\hline Previous palliative endocrine treatment & 46 & 13 & 33 & \\
\hline Tamoxifen only & $1(2.2)$ & $1(7.7)$ & 0 & 0.167 \\
\hline Letrozole & $23(50.0)$ & $7(53.8)$ & $16(48.5)$ & \\
\hline Tamoxifen and letrozole & $18(39.1)$ & $3(23.1)$ & $15(45.5)$ & \\
\hline Others & $4(8.7)$ & $2(15.4)$ & $2(6.1)$ & \\
\hline \multicolumn{5}{|l|}{ Disease status } \\
\hline Initially metastatic & $22(25.0)$ & $13(25.5)$ & $9(24.3)$ & $>0.99$ \\
\hline Recurrent & $66(75.0)$ & $38(74.5)$ & $28(75.7)$ & \\
\hline Disease-free interval $^{\text {a) }}$ & 66 & 38 & 28 & \\
\hline$\leq 24 \mathrm{mo}$ & $9(13.6)$ & $5(13.2)$ & $4(14.3)$ & $>0.99$ \\
\hline$>24 \mathrm{mo}$ & $57(86.4)$ & $33(86.8)$ & $24(85.7)$ & \\
\hline \multicolumn{5}{|l|}{ Previous endocrine resistance ${ }^{\mathrm{b})}$} \\
\hline Endocrine-sensitive & $58(65.9)$ & $36(70.6)$ & $22(59.5)$ & 0.030 \\
\hline Endocrine-resistant & $22(25.0)$ & $7(13.7)$ & $15(40.5)$ & \\
\hline No endocrine treatment & $8(9.1)$ & $8(15.7)$ & 0 & \\
\hline \multicolumn{5}{|l|}{ Previous palliative chemotherapy } \\
\hline Exposed & $19(21.6)$ & $4(7.8)$ & $15(40.5)$ & 0.001 \\
\hline Unexposed & $69(78.4)$ & $47(92.2)$ & $22(59.5)$ & \\
\hline \multicolumn{5}{|l|}{ ER/PR status } \\
\hline ER+ & $88(100)$ & $51(100)$ & $37(100)$ & - \\
\hline $\mathrm{PR}+$ & $54(61.4)$ & $32(62.7)$ & $22(59.5)$ & 0.928 \\
\hline \multicolumn{5}{|l|}{ No. of metastatic sites } \\
\hline 1 & $29(33.0)$ & $17(33.3)$ & $12(32.4)$ & 0.663 \\
\hline 2 & $30(34.1)$ & $19(37.3)$ & $11(29.7)$ & \\
\hline$\geq 3$ & $29(33.0)$ & $15(29.4)$ & $14(37.8)$ & \\
\hline \multicolumn{5}{|l|}{ Sites of metastases } \\
\hline Bone & $63(71.6)$ & $34(66.7)$ & $29(78.4)$ & 0.335 \\
\hline Bone only & $15(17.0)$ & $8(15.7)$ & $7(18.9)$ & 0.912 \\
\hline Visceral & $66(75.0)$ & $40(78.4)$ & $26(70.3)$ & 0.533 \\
\hline
\end{tabular}

(Continued to the next page) 
Table 1. Continued

\begin{tabular}{|c|c|c|c|c|}
\hline & Overall $(n=88)$ & $C \rightarrow E(n=51)$ & $E \rightarrow C(n=37)$ & p-value \\
\hline \multicolumn{5}{|l|}{ Treatment regimen } \\
\hline Everolimus+exemestane & $88(100)$ & $51(100)$ & $37(100)$ & - \\
\hline Palbociclib+letrozole & $40(45.5)$ & $40(78.4)$ & 0 & $<0.001$ \\
\hline Palbociclib+fulvestrant & $47(53.4)$ & $11(21.6)$ & $36(97.3)$ & \\
\hline Abemaciclib+fulvestrant & $1(1.1)$ & 0 & $1(2.7)$ & \\
\hline
\end{tabular}

Values are presented as median (range) or number (\%). C, cyclin-dependent kinase 4/6 inhibitor; E, everolimus; ECOG PS, Eastern

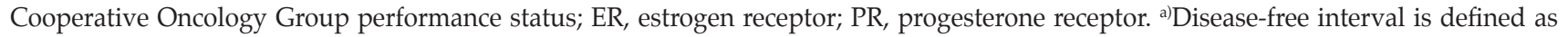
time from surgery to recurrence; or if not surgically treated, from initial diagnosis to the first date of confirmation of metastatic disease. Patients with initially metastatic disease were excluded, b) Endocrine sensitivity is defined as (1) at least 24 months of adjuvant endocrine therapy before recurrence, or (2) at least 24 weeks of palliative endocrine therapy for advanced disease.

entire study population, $53(60.2 \%)$ received CDK4/6i- or EVE-based regimens sequentially (34 [66.7\%] and 19 [51.4\%] in the $\mathrm{C} \rightarrow \mathrm{E}$ group and the $\mathrm{E} \rightarrow \mathrm{C}$ group, respectively), and the remaining 35 received endocrine treatment and/or chemotherapy in between (14 [27.5\%] in the $\mathrm{C} \rightarrow \mathrm{E}$ group and five [13.5\%] in the $\mathrm{E} \rightarrow \mathrm{C}$ group received endocrine treatment; four [7.8\%] in the $\mathrm{C} \rightarrow \mathrm{E}$ group and $14[37.8 \%]$ in the $\mathrm{E} \rightarrow \mathrm{C}$ group received chemotherapy).

\section{Survival outcomes}

Survival outcomes are shown in Fig. 2. With a median follow-up duration of 33.7 months (95\% confidence interval [CI], 31.5 to 35.2$)$, the median OS in the entire study population was 46.8 months (95\% CI, 38.9 to not estimated [NE]). No statistically significant differences were observed between groups, although the median $\mathrm{OS}$ of the $\mathrm{C} \rightarrow \mathrm{E}$ group was numerically longer than that of the $\mathrm{E} \rightarrow \mathrm{C}$ group (46.8 months [95\% CI, NE] vs. 38.9 months [95\% CI, 28.7 to NE], $\mathrm{p}=0.151$ ). Composite $\mathrm{PFS}_{\mathrm{C}+\mathrm{E}}$ throughout the CDK4/6i- and EVE-based treatments did not show significant differences between groups (median PFS $_{\mathrm{C}+\mathrm{E}}, 24.8$ months [95\% CI, 19.8 to 27.9 ] in the $\mathrm{C} \rightarrow \mathrm{E}$ group vs. 21.8 months [95\% CI, 16.3 to $28.1]$ in the $E \rightarrow C$ group; $p=0.681$ ). In the subgroup analysis stratified for clinical factors including endocrine resistance, presence of visceral metastases, line of treatment, and previous chemotherapy, there was no statistically significant composite $\mathrm{PFS}_{\mathrm{C}+\mathrm{E}}$ benefit due to treatment sequence for any subgroup (S1 Fig.).

Regarding the PFS for each regimen, $\mathrm{PFS}_{\mathrm{C}}$ was longer in the $\mathrm{C} \rightarrow \mathrm{E}$ group (median $\mathrm{PFS}_{\mathrm{C}}, 13.4$ months [95\% CI, 8.4 to 14.8 ] vs. 4.8 months [95\% CI, 3.4 to 6.3 ], $\mathrm{p}<0.001$ ), whereas $\mathrm{PFS}_{\mathrm{E}}$ was not significantly different between groups, although it tended to be longer in the $\mathrm{E} \rightarrow \mathrm{C}$ group (median $\mathrm{PFS}_{\mathrm{E}}$, 6.0 months [ $95 \% \mathrm{CI}, 4.8$ to 8.0 ] vs. 8.4 months [ $95 \% \mathrm{CI}, 6.8$ to 11.6], $\mathrm{p}=0.224)$. As for the PFS by treatment sequence, neither $\mathrm{PFS}_{1}$ nor $\mathrm{PFS}_{2}$ differed significantly between groups, although both tended to be longer in the $\mathrm{C} \rightarrow \mathrm{E}$ group (median $\mathrm{PFS}_{1}, 13.4$ months [95\% CI, 8.4 to 14.8 ] vs. 8.4 months [95\% CI, 6.8 to 11.6], $\mathrm{p}=0.286$; median $\mathrm{PFS}_{2}, 6.0$ months [95\% CI, 4.8 to 8.0 ] vs. 4.8 months [ $95 \%$ CI, 3.4 to 6.3 ], $\mathrm{p}=0.132$ ). Similar results were observed in the subgroup of patients who received both regimens as second-line treatment and above (18 patients in the $\mathrm{C} \rightarrow \mathrm{E}$ group, 33 patients in the $\mathrm{E} \rightarrow \mathrm{C}$ group) (S2 Fig.).

The duration of treatment is shown in a swimmer plot (Fig. 3). Overall, $\mathrm{PFS}_{2}$ tended to be shorter than $\mathrm{PFS}_{1}$ in both groups. When comparing $\mathrm{PFS}_{2}$ with $\mathrm{PFS}_{1}$ for patients who were followed up until discontinuation of both regimens, the median $\mathrm{PFS}_{2} / \mathrm{PFS}_{1}$ ratio did not differ significantly between groups (0.5 [interquartile range (IQR), 0.3 to 1.3 ] in the $\mathrm{C} \rightarrow \mathrm{E}$ group, 0.6 [IQR, 0.3 to 1.1] in the $\mathrm{E} \rightarrow \mathrm{C}$ group, $\mathrm{p}=0.775)$.

\section{Tumor response}

Tumor response was assessed by RECIST ver. 1.1 in patients who had measurable disease and had at least one tumor response evaluation by radiologic imaging (Table 2). The overall response rate (ORR) to the preceding regimen tended to be higher; ORR to the CDK4/6i-based regimen was $48.6 \%$ in the $\mathrm{C} \rightarrow \mathrm{E}$ group but $22.6 \%$ in the $\mathrm{E} \rightarrow \mathrm{C}$ group $(\mathrm{p}=0.049)$. Conversely, the ORR to the EVE-based regimen was $9.5 \%$ in the $\mathrm{C} \rightarrow \mathrm{E}$ group and $20.0 \%$ in the $\mathrm{E} \rightarrow \mathrm{C}$ group $(\mathrm{p}=0.302)$.

\section{Subsequent treatment}

At data cutoff, 39 patients $(76.5 \%)$ in the $\mathrm{C} \rightarrow \mathrm{E}$ group and $26(70.3 \%)$ in the $\mathrm{E} \rightarrow \mathrm{C}$ group discontinued both regimens. For CDK4/ 6 inhibitors, the reason for discontinuing treatment was disease progression in all patients; for EVE, 93.4\% of the patients discontinued treatment due to disease progression (Fig. 1). Ten patients (11.4\%) discontinued EVE during EVE-based treatment and were treated with exemestane only, all because of adverse events, with seven patients expe- 
A
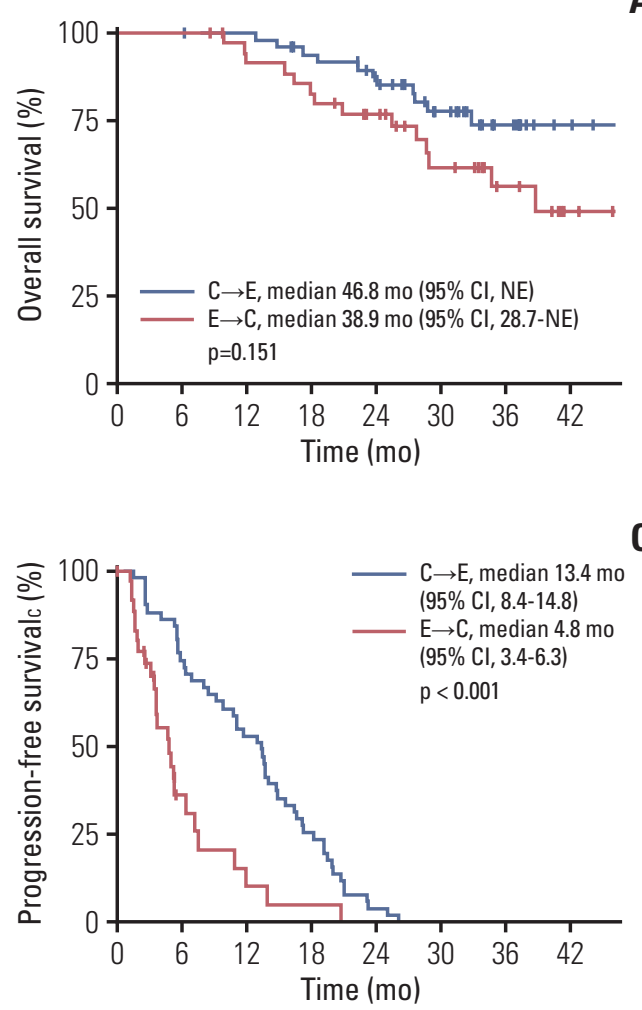

E

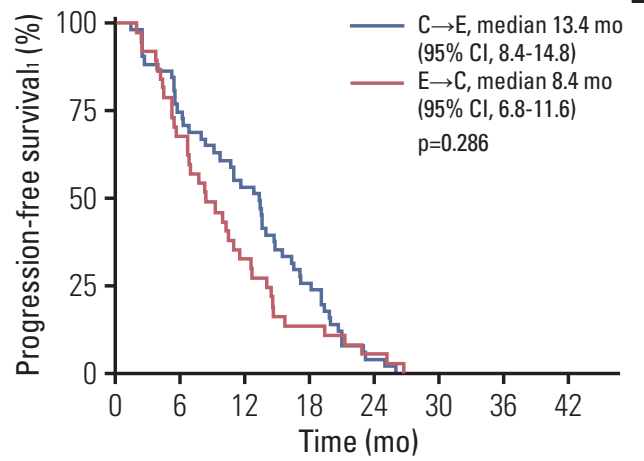

C
B
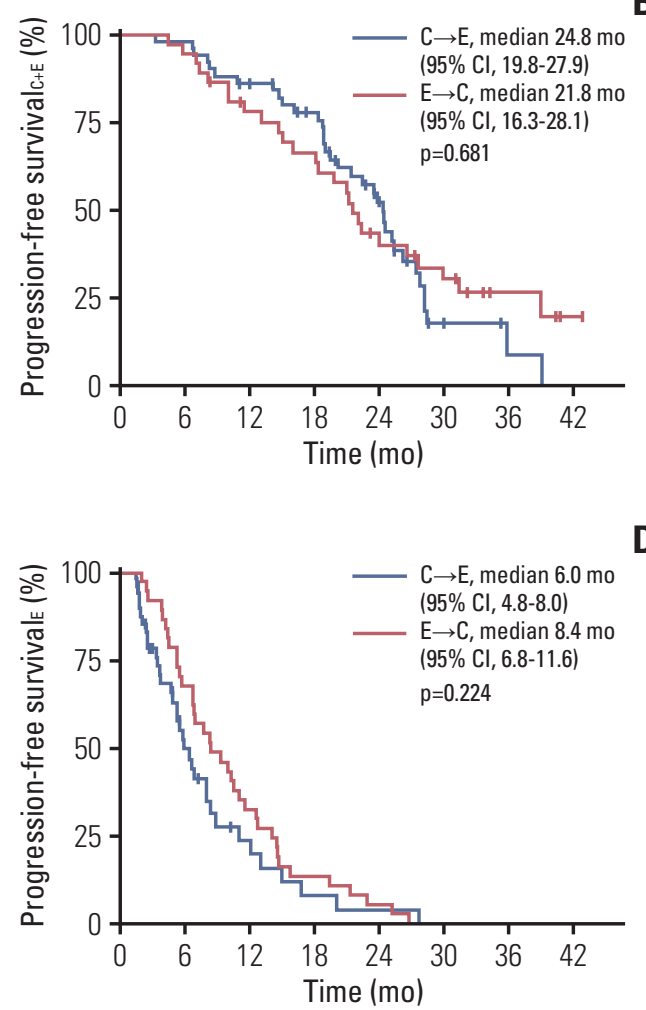

$\mathbf{F}$

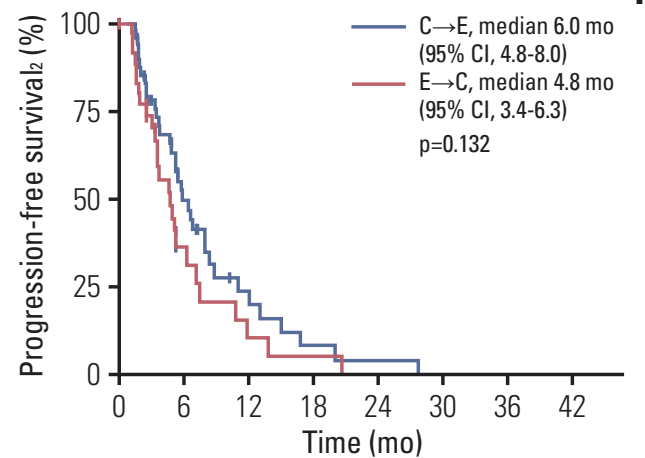

Fig. 2. Survival outcomes. (A) Overall survival from the start of preceding regimen. (B) Progression-free survival $\mathrm{C}+\mathrm{E}_{\mathrm{E}}$, composite progression-free survival from the start of preceding regimen to the event of the following regimen between CDK4/6i or EVE-based regimens. (C) Progression-free survivalc, progression-free survival for CDK4/ 6i-based regimen. (D) Progression-free survivale, progression-free survival for EVE-based regimen. (E) Progression-free survival, progression-free survival for the preceding regimen and (F) progression-free survival$_{2}$, progression-free survival for the following regimen between CDK4/6i- and EVE-based regimens. C or CDK4/6i, cyclin-dependent kinase 4/ 6 inhibitor; CI, confidence interval; E or EVE, everolimus; NE, not estimated.

riencing pneumonitis. However, two patients $(2.3 \%)$ discontinued the CDK4/6i during CDK4/6i-based treatment and continued endocrine treatment only; one patient experienced an adverse event (fatigue), and one patient cited economic problems. The median time from the start of either regimen to the start of subsequent palliative chemotherapy was 23.0 months (95\% CI, 15.4 to 25.2 ) in the $\mathrm{C} \rightarrow \mathrm{E}$ group and 11.5 months ( $95 \%$ CI, 7.8 to 18.4 ) in the $\mathrm{E} \rightarrow \mathrm{C}$ group $(\mathrm{p}=0.061)$. 

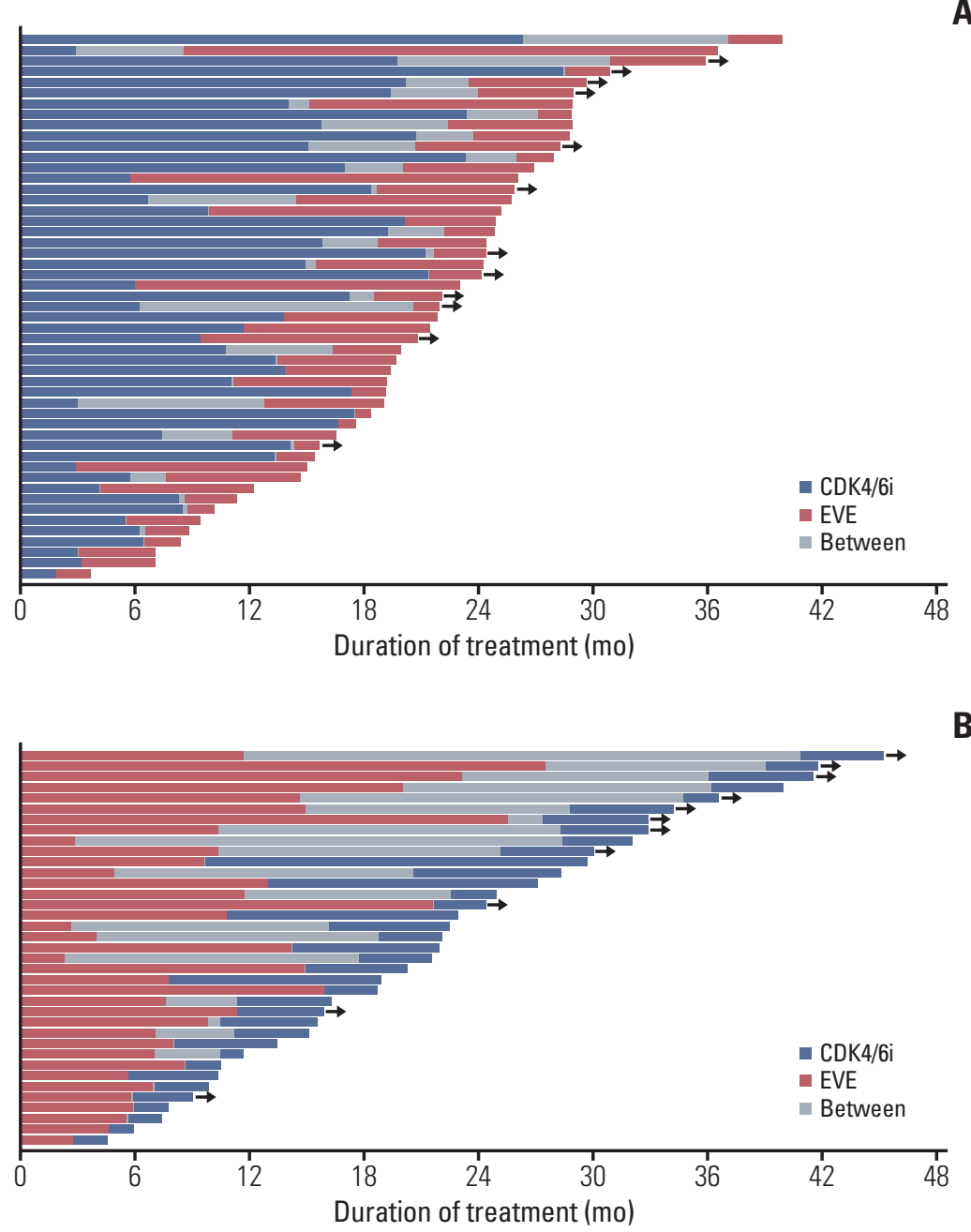

Fig. 3. Swimmer plot for treatment duration. (A) $C \rightarrow$ E group. (B) $E \rightarrow C$ group. C or CDK4/ 6 i, cyclin-dependent kinase $4 / 6$ inhibitors; E or EVE, everolimus. Black arrows indicate ongoing treatment.

\section{Discussion}

In this retrospective study in which clinical outcomes based on treatment sequence were assessed between CDK4/6i- and EVE-based regimens for HR+ HER2- MBC, survival outcomes including $\mathrm{PFS}_{\mathrm{C}+\mathrm{E}}, \mathrm{PFS}_{\mathrm{C}}, \mathrm{PFS}_{1}, \mathrm{PFS}_{2}$, and OS tended to favor the $\mathrm{C} \rightarrow \mathrm{E}$ group, but only $\mathrm{PFS}_{\mathrm{C}}$ showed statistically significant differences between groups. Survival outcomes shown in this study were consistent with prior results on CDK4/6i- or EVE-based treatments [11,17], with PFS of 8.4-13.4 months to the preceding regimen. PFS to the following treatment tended to be shorter than that of preceding regimens across the study population, represented by $\mathrm{PFS}_{2} / \mathrm{PFS}_{1}$ ratios less than 1 in both groups. A similar tendency was noted in tumor response, with higher ORRs to the preceding regimen.
The result of this study, including the survival outcomes, tumor responses, times to chemotherapy, and the rates of treatment discontinuation, favored the $\mathrm{C} \rightarrow \mathrm{E}$ group over the $\mathrm{E} \rightarrow \mathrm{C}$ group. However, there were imbalances between the two groups; patients included in the $\mathrm{E} \rightarrow \mathrm{C}$ group were more heavily treated, with a higher proportion of endocrine-resistant disease and prior exposure to chemotherapy. It is noteworthy that despite these imbalances in baseline characteristics, survival outcomes, including composite $\mathrm{PFS}_{\mathrm{C}+\mathrm{E}}$, did not result in statistically significant differences between groups. Although further study is needed to confirm these findings and tell the sequence of these two agents may have little impact on the long-term survival outcome of HR+ HER2MBC in which a long journey of treatment is expected, these results suggest that CDK4/ 6i- and EVE-based treatments can be valid treatment options in circumstances where the other 
Table 2. Tumor response

\begin{tabular}{|c|c|c|c|}
\hline & $\mathrm{C} \rightarrow \mathrm{E}$ & $\mathbf{E} \rightarrow \mathrm{C}$ & p-value \\
\hline Best overall response to $\mathrm{CDK} 4 / 6$ inhibitor-based regimen & Measurable $(\mathrm{n}=37)$ & Measurable $(\mathrm{n}=32)$ & \\
\hline Partial response & $18(48.6)$ & $7(21.9)$ & \\
\hline Stable disease & $15(40.5)$ & $13(40.6)$ & \\
\hline Progressive disease & $4(10.8)$ & $11(34.4)$ & \\
\hline Not evaluated & 0 & $1(3.1)^{a)}$ & \\
\hline Best overall response to EVE-based regimen & Measurable $(\mathrm{n}=44)$ & Measurable $(\mathrm{n}=30)$ & \\
\hline Partial response & $4(9.1)$ & $6(20.0)$ & \\
\hline Stable disease & $28(63.6)$ & $20(66.7)$ & \\
\hline Progressive disease & $10(22.7)$ & $4(13.3)$ & \\
\hline Not evaluated & $2(4.5)$ & 0 & \\
\hline \multicolumn{4}{|l|}{ Overall response rate ${ }^{\mathrm{b})}$} \\
\hline Overall response rate to EVE & $4 / 42(9.5)$ & $6 / 30(20.0)$ & 0.302 \\
\hline Overall response rate to CDK4/6 inhibitor & $18 / 37(48.6)$ & $7 / 31(22.6)$ & 0.049 \\
\hline
\end{tabular}

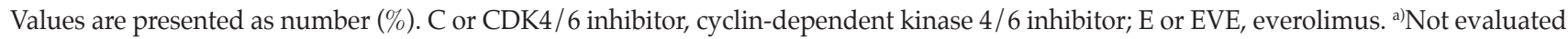
due to the loss of follow-up, ${ }^{b}$ Including patients with measurable disease, and had at least one tumor response evaluation result. All best objective responses for objective response rate were partial responses.

treatment had been already given.

Nonetheless, CDK4/6i-based treatment is now widely accepted as a standard treatment choice in HR+ HER2- MBC patients [18] and should be considered first. Not only do CDK4 / 6i provide an OS benefit, but they also have a favorable adverse event profile compared with EVE $[11,19]$. Consistent with previous studies, our study showed a higher rate of treatment discontinuation for EVE than for CDK4/6i during treatment ( $11 \%$ vs. $2 \%)$; of the patients who discontinued EVE, 70\% had developed pneumonitis. Therefore, the survival outcomes of our study suggest that EVE-based regimens could be a reasonable choice after CDK4/6i failure, in which the optimal treatment strategies have yet to be established. These results are in line with prior retrospective studies that have reported similar PFS with EVE between CDK4/ 6i-exposed and unexposed patients [20], and a modest survival outcome with a median PFS of four months and a median OS of 19 months with EVE following CDK4/ $6 \mathrm{i}$ in a heavily treated patient population [21].

Meanwhile, the results of the retrospective studies that evaluated the efficacy of CDK4/ 6i following EVE are contradictory. A small retrospective study of 23 patients, of whom 95\% were treated with $\geq 3$ lines of endocrine treatment, reported an ORR of $0 \%$ and a median PFS of 2.9 months with palbociclib following EVE [22]. However, other studies that included larger numbers of patients have reported no differences in the efficacy of palbociclib after previous EVE treatment, with median time-to-treatment failure or PFS of approximately 5-6 months [23,24], similar to the median $\mathrm{PFS}_{\mathrm{C}}$ in the $\mathrm{E} \rightarrow \mathrm{C}$ group of this study (4.8 months). Although the $\mathrm{PFS}_{\mathrm{C}}$ was shorter in the $\mathrm{E} \rightarrow \mathrm{C}$ group than in the $\mathrm{C} \rightarrow \mathrm{E}$ group, the clinically meaningful $\mathrm{PFS}_{\mathrm{C}}$ and the comparable long-term survival outcomes between groups in our study suggests that CDK4/6i could also be a useful treatment option in patients with prior exposure to EVE.

This study is limited by its single-center retrospective nature. A considerable proportion of patients were on treatment at the data cutoff, which resulted in a shorter followup duration for the second regimen. Similarly, the clinical benefit rate could not be assessed because of differences in the follow-up durations between preceding and following treatments. The effects of unmeasured confoundings, including treatments other than CDK4/ 6i- or EVE-based regimens, cannot be eliminated. In particular, a significant portion of patients in cases for which CDK4/6i were unaffordable for the first-line treatment was expected to receive endocrine treatment before $\mathrm{CDK} 4 / 6 \mathrm{i}$ were added to the treatment. This could adversely affect the treatment outcomes of the CDK4/6i [25]. Lastly, imbalances in the baseline characteristics should be considered when interpreting the results. However, the lack of statistically significant differences in the major survival outcomes, despite the unfavorable clinical characteristics of the $\mathrm{E} \rightarrow \mathrm{C}$ group, further supports the results of this study.

In conclusion, although the CDK4/6i-based regimen should be considered as an earlier line of treatment given the favorable survival outcomes, tumor responses, times to subsequent chemotherapy, and reduced treatment discontinuation related to toxicity, CDK4/6i- and EVE-based treatments can be valid options in circumstances where the other treatment had been already given. 
Electronic Supplementary Material

Supplementary materials are available at Cancer Research and Treatment website (https:// www.e-crt.org).

\section{Ethical Statement}

This study was approved by the Institutional Review Board of the Asan Medical Center (\#2021-0111) and conducted in accordance with the Declaration of Helsinki. The IRB granted an informed-consent waiver for this retrospective study.

\section{Author Contributions}

Conceived and designed the analysis: Kim SB.

Collected the data: Jeong $\mathrm{H}$.

Contributed data or analysis tools: Jeong JH, Kim JE, Ahn JH, Jung
KH.

Performed the analysis: Jeong $\mathrm{H}$.

Wrote the paper: Jeong $\mathrm{H}$, Jeong JH.

\section{Conflicts of Interest}

$\mathrm{KHJ}$ reports personal fees from AstraZeneca, personal fees from Roche, personal fees from Celgene, personal fees from Novartis, personal fees from Takeda, outside the submitted work.

S-BK reports research funding from Novartis, Sanofi-Aventis and DongKook Pharm Co. and has participated as a consultant in advisory boards for Novartis, AstraZeneca, Lilly, Enzychem, Dae Hwa Pharmaceutical Co. Ltd, ISU Abxis, and Daiichi-Sankyo, outside the submitted work. All remaining authors have declared no conflicts of interest.

\section{References}

1. Finn RS, Martin M, Rugo HS, Jones S, Im SA, Gelmon K, et al. Palbociclib and letrozole in advanced breast cancer. N Engl J Med. 2016;375:1925-36.

2. Goetz MP, Toi M, Campone M, Sohn J, Paluch-Shimon S, Huober J, et al. MONARCH 3: abemaciclib as initial therapy for advanced breast cancer. J Clin Oncol. 2017;35:3638-46.

3. Hortobagyi GN, Stemmer SM, Burris HA, Yap YS, Sonke GS, Paluch-Shimon S, et al. Ribociclib as first-line therapy for HRpositive, advanced breast cancer. N Engl J Med. 2016;375:173848.

4. Turner NC, Ro J, Andre F, Loi S, Verma S, Iwata H, et al. Palbociclib in hormone-receptor-positive advanced breast cancer. N Engl J Med. 2015;373:209-19.

5. Sledge GW Jr, Toi M, Neven P, Sohn J, Inoue K, Pivot X, et al. MONARCH 2: abemaciclib in combination with fulvestrant in women with HR+/HER2- advanced breast cancer who had progressed while receiving endocrine therapy. J Clin Oncol. 2017;35:2875-84

6. Slamon DJ, Neven P, Chia S, Fasching PA, De Laurentiis M, Im SA, et al. Phase III randomized study of ribociclib and fulvestrant in hormone receptor-positive, human epidermal growth factor receptor 2-negative advanced breast cancer: MONALEESA-3. J Clin Oncol. 2018;36:2465-72.

7. Tripathy D, Im SA, Colleoni M, Franke F, Bardia A, Harbeck $\mathrm{N}$, et al. Ribociclib plus endocrine therapy for premenopausal women with hormone-receptor-positive, advanced breast cancer (MONALEESA-7): a randomised phase 3 trial. Lancet Oncol. 2018;19:904-15

8. Sledge GW Jr, Toi M, Neven P, Sohn J, Inoue K, Pivot X, et al. The effect of abemaciclib plus fulvestrant on overall survival in hormone receptor-positive, ERBB2-negative breast cancer that progressed on endocrine therapy-MONARCH 2: a randomized clinical trial. JAMA Oncol. 2020;6:116-24.

9. Im SA, Lu YS, Bardia A, Harbeck N, Colleoni M, Franke F, et al. Overall survival with ribociclib plus endocrine therapy in breast cancer. N Engl J Med. 2019;381:307-16.

10. Slamon DJ, Neven P, Chia S, Fasching PA, De Laurentiis M, Im $\mathrm{SA}$, et al. Overall survival with ribociclib plus fulvestrant in advanced breast cancer. N Engl J Med. 2020;382:514-24.

11. Baselga J, Campone M, Piccart M, Burris HA 3rd, Rugo HS, Sahmoud T, et al. Everolimus in postmenopausal hormonereceptor-positive advanced breast cancer. N Engl J Med. 2012;366:520-9.

12. Piccart M, Hortobagyi GN, Campone M, Pritchard KI, Lebrun $\mathrm{F}$, Ito $\mathrm{Y}$, et al. Everolimus plus exemestane for hormonereceptor-positive, human epidermal growth factor receptor2-negative advanced breast cancer: overall survival results from BOLERO-2dagger. Ann Oncol. 2014;25:2357-62.

13. Jansen VM, Bhola NE, Bauer JA, Formisano L, Lee KM, Hutchinson $\mathrm{KE}$, et al. Kinome-wide RNA interference screen reveals a role for PDK1 in acquired resistance to CDK4/6 inhibition in ER-positive breast cancer. Cancer Res. 2017;77:2488-99.

14. Herrera-Abreu MT, Palafox M, Asghar U, Rivas MA, Cutts RJ, Garcia-Murillas I, et al. Early adaptation and acquired resistance to CDK4/6 inhibition in estrogen receptor-positive breast cancer. Cancer Res. 2016;76:2301-13.

15. Yamamoto T, Kanaya N, Somlo G, Chen S. Synergistic anticancer activity of CDK4/6 inhibitor palbociclib and dual mTOR kinase inhibitor MLN0128 in pRb-expressing ER-negative breast cancer. Breast Cancer Res Treat. 2019;174:615-25.

16. Michaloglou C, Crafter C, Siersbaek R, Delpuech O, Curwen JO, Carnevalli LS, et al. Combined inhibition of mTOR and CDK4/6 is required for optimal blockade of E2F function and long-term growth inhibition in estrogen receptor-positive breast cancer. Mol Cancer Ther. 2018;17:908-20.

17. Cristofanilli M, Turner NC, Bondarenko I, Ro J, Im SA, Masuda N, et al. Fulvestrant plus palbociclib versus fulvestrant plus placebo for treatment of hormone-receptor-positive, HER2-negative metastatic breast cancer that progressed on previous endocrine therapy (PALOMA-3): final analysis of 
the multicentre, double-blind, phase 3 randomised controlled trial. Lancet Oncol. 2016;17:425-39.

18. Cardoso F, Paluch-Shimon S, Senkus E, Curigliano G, Aapro MS, Andre F, et al. 5th ESO-ESMO international consensus guidelines for advanced breast cancer (ABC 5). Ann Oncol. 2020;31:1623-49.

19. Chirila C, Mitra D, Colosia A, Ling C, Odom D, Iyer S, et al. Comparison of palbociclib in combination with letrozole or fulvestrant with endocrine therapies for advanced/metastatic breast cancer: network meta-analysis. Curr Med Res Opin. 2017;33:1457-66.

20. Cook MM, Al Rabadi L, Kaempf AJ, Saraceni MM, Savin MA, Mitri ZI. Everolimus plus exemestane treatment in patients with metastatic hormone receptor-positive breast cancer previously treated with CDK4/6 inhibitor therapy. Oncologist. 2021;26:101-6.

21. Dhakal A, Antony Thomas R, Levine EG, Brufsky A, Takabe K, Hanna MG, et al. Outcome of everolimus-based therapy in hormone-receptor-positive metastatic breast cancer pa- tients after progression on palbociclib. Breast Cancer (Auckl). 2020;14:1178223420944864.

22. Dhakal A, Matthews CM, Levine EG, Salerno KE, Zhang F, Takabe K, et al. Efficacy of palbociclib combinations in hormone receptor-positive metastatic breast cancer patients after prior rverolimus yreatment. Clin Breast Cancer. 2018;18:e14015.

23. du Rusquec P, Palpacuer C, Campion L, Patsouris A, Augereau P, Gourmelon C, et al. Efficacy of palbociclib plus fulvestrant after everolimus in hormone receptor-positive metastatic breast cancer. Breast Cancer Res Treat. 2018;168:559-66.

24. Kovac A, Kuhar CG, Ovcaricek T, Matos E, Mencinger M, Borstnar S. Efficacy of palbociclib after everolimus in hormone receptor-positive, HER2-negative advanced breast cancer. J Clin Oncol. 2020;38(15_Suppl):e13030.

25. O'Leary B, Cutts RJ, Liu Y, Hrebien S, Huang X, Fenwick K, et al. The genetic landscape and clonal evolution of breast cancer resistance to palbociclib plus fulvestrant in the PALOMA-3 trial. Cancer Discov. 2018;8:1390-403. 